\title{
Opalski Syndrome Caused by Vertebral Artery Compression of the Lateral Surface of the Medulla Oblongata
}

\author{
Tomohisa Dembo ${ }^{1,2}$ and Norio Tanahashi ${ }^{2}$
}

\begin{abstract}
A 55-year-old man presented with vertigo, nystagmus, and gait ataxia followed by left hemiparesis (Opalski syndrome). T2-weighted magnetic resonance imaging revealed vascular compression of the left lateral side of the medulla oblongata by the left vertebral artery. On diffusion tensor imaging, the level of fractional anisotropy (FA) in the left corticospinal fibers caudal to the pyramidal decussation was lower than that observed in the right corticospinal fibers. Opalski syndrome caused by vascular compression is very rare. This is the first reported case of Opalski syndrome that was imaged on FA.
\end{abstract}

Key words: Opalski syndrome, ipsilateral hemiparesis, lateral medullary syndrome, vertebral artery compression, diffusion tensor imaging, fractional anisotrophy

(Intern Med 52: 1115-1120, 2013)

(DOI: 10.2169/internalmedicine.52.7177)

\section{Introduction}

In 1946, Opalski reported two patients with ipsilateral facial loss of pain and temperature sensation, Horner syndrome, hemiplegia, ataxia, and contralateral loss of pain and temperature sensation in the limbs (1). Opalski syndrome is considered to be a variant of a lateral medulla oblongata infarct (i.e., Wallenberg syndrome) with ipsilateral hemiplegia (2). Some pathological studies and neuroradiological findings obtained with conventional magnetic resonance imaging (MRI) have shown that lesions causing this syndrome are located caudal to the lesions in Wallenberg syndrome and involve the corticospinal fibers caudal to the pyramidal decussation (3).

Thus far, most described causes of this syndrome have been reported to result from vertebral artery (VA) occlusion/ stenosis and/or VA dissection (3, 5-7). Meanwhile, controversy remains whether the hemiparesis observed in atypical Wallenberg syndrome results from the extension of a lateral medullary infarct into the pyramidal tract or from hypotonic dysregulation of motor loops (8). MRI has only inconsis- tently shown pyramidal tract involvement in patients with Opalski syndrome (3-5, 9-11).

There are several reports concerning medulla oblongata dysfunction caused by vascular compression (12-18). We herein present a case of Opalski syndrome caused by vascular compression of the lateral side of the medulla oblongata by the VA, which was confirmed on fractional anisotropy (FA) mapping with diffusion tensor imaging. The present case report adds information regarding the anatomical basis of ipsilateral hemiparesis in lateral medullary syndrome and confirms Opalski's hypothesis, which suggests that the motor impairment observed in this disease is a result of the extension of the lesion from the lateral medulla oblongata to the upper cervical cord and involves corticospinal fibers caudal to the pyramidal decussation.

\section{Case Report}

In March 2006, a 55-year-old right-handed man experienced vertigo that spontaneously resolved within a few hours. In April 2006, he again suddenly developed vertigo that was followed by nausea and vomiting. His gait was un-

${ }^{1}$ Department of Neurology, EIJU General Hospital, Japan and ${ }^{2}$ Department of Neurology and Cerebrovascular Medicine, Saitama Medical University International Medical Center, Japan

Received for publication December 23, 2011; Accepted for publication January 20, 2013

Correspondence to Dr. Tomohisa Dembo, tomdembo-keio@umin.ac.jp 

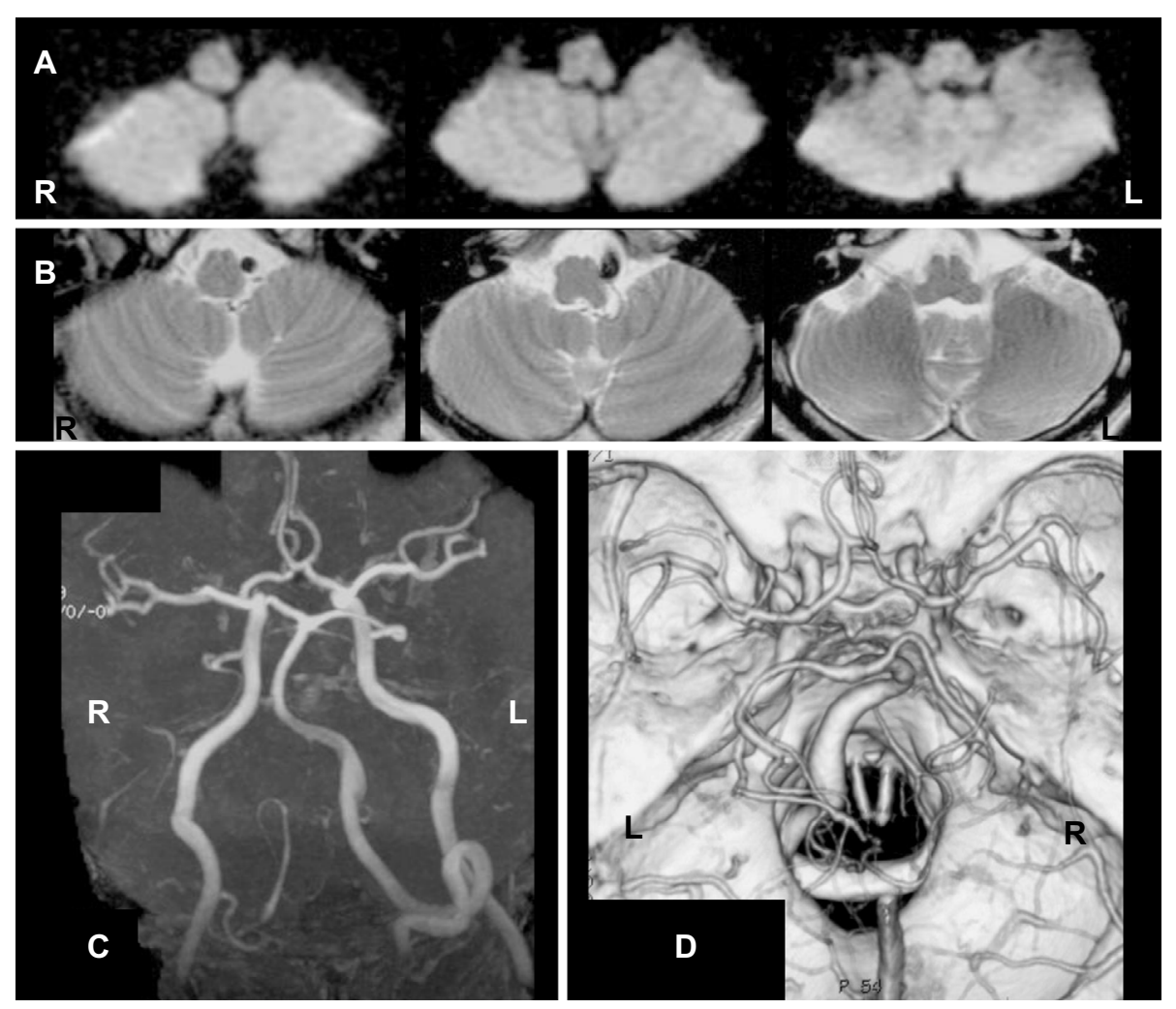

Figure 1. A. Magnetic resonance diffusion-weighted imaging obtained on admission revealed no evidence of cerebral infarction. B. T2-weighted magnetic resonance imaging obtained on admission revealed a flow-void area representing the left vertebral artery compressing and distorting the left lateral surface of the medulla oblongata. C, D. Magnetic resonance angiography (C) and 3-dimensional computed tomography angiography (D) performed on admission revealed a tortuous and dolichoectatic left vertebral artery, findings suggestive of arterial dissection.

steady and he could not stand without holding onto a support. He was taken to the emergency department of our hospital by ambulance. He did not complain of headaches, posterior cervical pain, tinnitus, hiccups, hoarseness or numbness. He was otherwise healthy and had not been diagnosed with hypertension prior to that time. There was neither a history of recent neck trauma or chiropractic treatment nor a family history of stroke. He was admitted to our institute.

On admission, hypertension $(164 / 104 \mathrm{mmHg})$ was noted on physical examination. The patient was alert. A neurological examination disclosed vertigo, nystagmus, and gait ataxia; however, no dysphagia, deviation of the palate or tongue, Horner syndrome, sensory impairment, limb ataxia or hemiparesis were observed. The patient's clinical findings were suggestive of atypical left lateral medullary syndrome (i.e., Wallenberg syndrome).

Emergent MRI diffusion-weighted imaging (DWI) revealed no evidence of cerebral infarction. T2-weighted magnetic resonance imaging (T2WI) revealed a flow-void area that corresponded to the left VA compressing and distorting the left lateral surface of the medulla oblongata. Magnetic resonance angiography (MRA) and 3-dimensional computed tomography angiography revealed a tortuous and dolichoectatic left VA. These findings were suggestive of arterial dissection (Fig. 1). The patient's treatment was conservative and consisted primarily of management of hypertension.

Four days after admission, he developed left hemiparesis. On the left side, the tendon reflexes were exaggerated and a pathological plantar reflex (positive Babinski sign) was elicited. The grade of paralysis on the left side was slightly more severe in the leg than in the arm. Emergent MRI T2 WI of the cervical and lumbar spine revealed that the only significant finding was slight protrusion of the cervical (C34, C4-5, C5-6, C6-7, and C7-8) and lumbar (L2-3, L3-4, and L4-5) discs (Fig. 2). Although slight protrusion of the cervical and lumbar discs was present in this case, any contribution of the cervical and lumbar disc lesion to the hemiparesis on the left side was considered to be insignificant. Emergent MRI of the head was then performed again. DWI revealed no evidence of cerebral infarction; however, T2WI revealed severe vascular compression of the left ventrolateral side of the lower medulla oblongata by the left VA. The medulla oblongata was kidney-shaped, with a deep indentation on the left side (Fig. 3). The level of FA in the left-sided corticospinal fibers caudal to the pyramidal decussation was lower than that observed in the corresponding right-sided corticospinal fibers (Fig. 4). This finding was interpreted as suggesting the contribution of vascular compression to the left hemiparesis. The patient received no specific treatment for this condition; however, stricter management of hyper- 

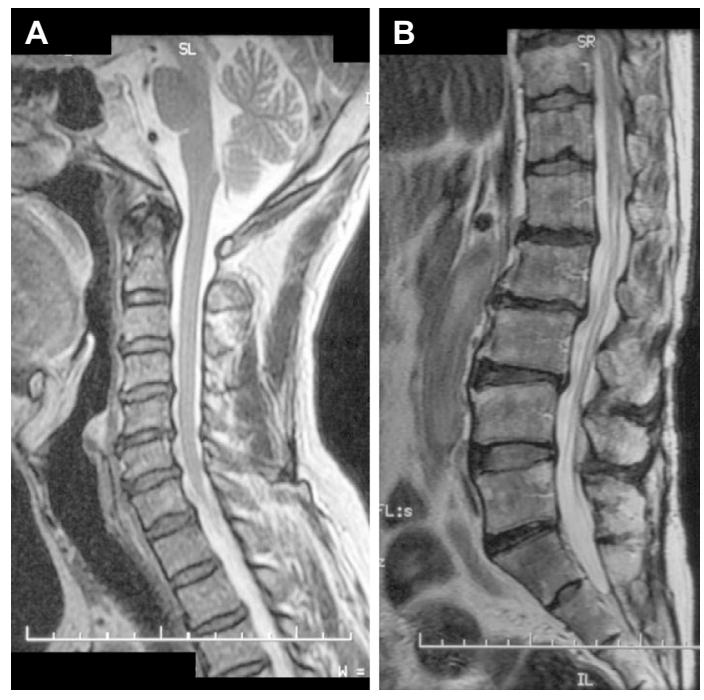

Figure 2. A, B. Magnetic resonance T2-weighted imaging of the cervical (A) and lumbar (B) spine at four days after admission revealed that the only significant finding was slight protrusion of the cervical (C3-4, C4-5, C5-6, C6-7, and C7-8) and lumbar (L2-3, L3-4, and L4-5) discs.

tension and rehabilitation was initiated. The patient's symptoms persisted for several weeks. Although decompression surgery was considered, the symptoms gradually improved and resolved within one month without therapeutic surgery. At that time, diffusion tensor imaging was performed again, and the decreased FA level previously seen in the left corticospinal fibers improved (Fig. 4). In June 2006, the patient was discharged. After discharge, he returned to his previous activities of daily living and has not required any antihypertensive drugs due to improvement in his hypertension. Follow-up MRI of the head in October 2006 revealed no evidence of cerebral infarction (Fig. 5). The patient has remained healthy for almost five years of follow-up.

\section{Discussion}

This patient presented with atypical left lateral medullary syndrome and ipsilateral hemiparesis (Opalski syndrome) caused by tortuous and dolichoectatic left VA compression of the left lateral surface of the medulla oblongata.

In his original description of the syndrome, Opalski suggested that the motor impairment was the result of extension of ischemia from the lateral medulla oblongata to the upper cervical cord involving corticospinal fibers caudal to the pyramidal decussation. He also suggested that the ischemia was related to occlusion of the posterior spinal artery (1). Subsequently, other authors have reported cases related to VA occlusion (4). Moreover, other authors have attributed these combined findings to VA occlusion/stenosis and/or VA dissection that compromise the medullary penetrating arteries arising from distal VAs or anterior spinal arteries $(3,5-7)$. Opalski syndrome may be due to either ischemic disturbances (such as distal VA dissection) or the effects of hemodynamically vulnerable areas such as the 'borderline zone' between the anterior and posterior spinal arteries or between the vertebral and spinal arteries $(5,7)$. Meanwhile, controversy remains as to whether hemiparesis in atypical Wallenberg syndrome results from extension of a lateral medullary infarct into the pyramidal tract or from hypotonic dysregulation of motor loops (8). In addition, MRI has so far inconsistently shown pyramidal tract involvement (3-5, 9-11).

Iwasaki et al. (17) described the case of a 54-year-old man who presented with hypoalgesia and hypothermesthesia (pain and thermal numbness) in the left lower extremity due to compression of the lateral side of the right medulla oblongata by an elongated right VA. They noted that the artery was thought to compress the lateral spinothalamic tract and that the patient's symptoms were provoked by abnormal compression of the VA. They concluded that compression of the medulla oblongata by the VA induced the medulla oblongata dysfunction that had presented with clinical atypical lateral medullary syndrome (17). In the present case, it was thought that a similar mechanism was responsible for the patient's atypical lateral medullary syndrome.

Few reports have so far described the phenomenon of the VA compressing the lateral side of the medulla oblongata and causing neurological deficits (such as pyramidal tract signs predominantly in the ipsilateral lower extremity). In 1985, Kim et al. (12) reported the first such case. They stated that, in the lateral corticospinal tract, after its decussation, the fibers that innervate the lower extremities are laterally located and those innervating the upper extremities are medially located. They concluded that the fact that paresis begins and is more pronounced in the leg corresponds well with the topographical distribution of these nerve fibers (12).

Furthermore, Ishikawa et al. (18) reported the case of a 37-year-old man who presented with Opalski syndrome. MR cisternography at the medulla oblongata disclosed marked compression and deformity of the medulla oblongata by a dolichoectatic right VA that had pushed the left VA aside. They said that the presence of Opalski syndrome in that patient could be attributed to vascular compression by the dolichoectatic VA. They mentioned that Opalski syndrome can be provoked not only by infarction, but also by medulla oblongata dysfunction caudal to the pyramidal decussation caused by vascular compression (18). Using diffusion tensor imaging in a patient with a lateral-medullary infarction, Nakamura et al. (9) indicated that involvement of the ipsilateral corticospinal tract following pyramidal decussation can cause Opalski syndrome. In the present case, when the patient presented with left hemiparesis, the FA level in the left corticospinal fibers caudal to the pyramidal decussation was decreased compared to that observed in the right corticospinal fibers. In contrast, when the symptoms resolved, the decreased FA level in the left corticospinal fibers improved. On diffusion tensor imaging, it has been shown that the FA level is an indicator of the strength of diffusion anisotrophy. 


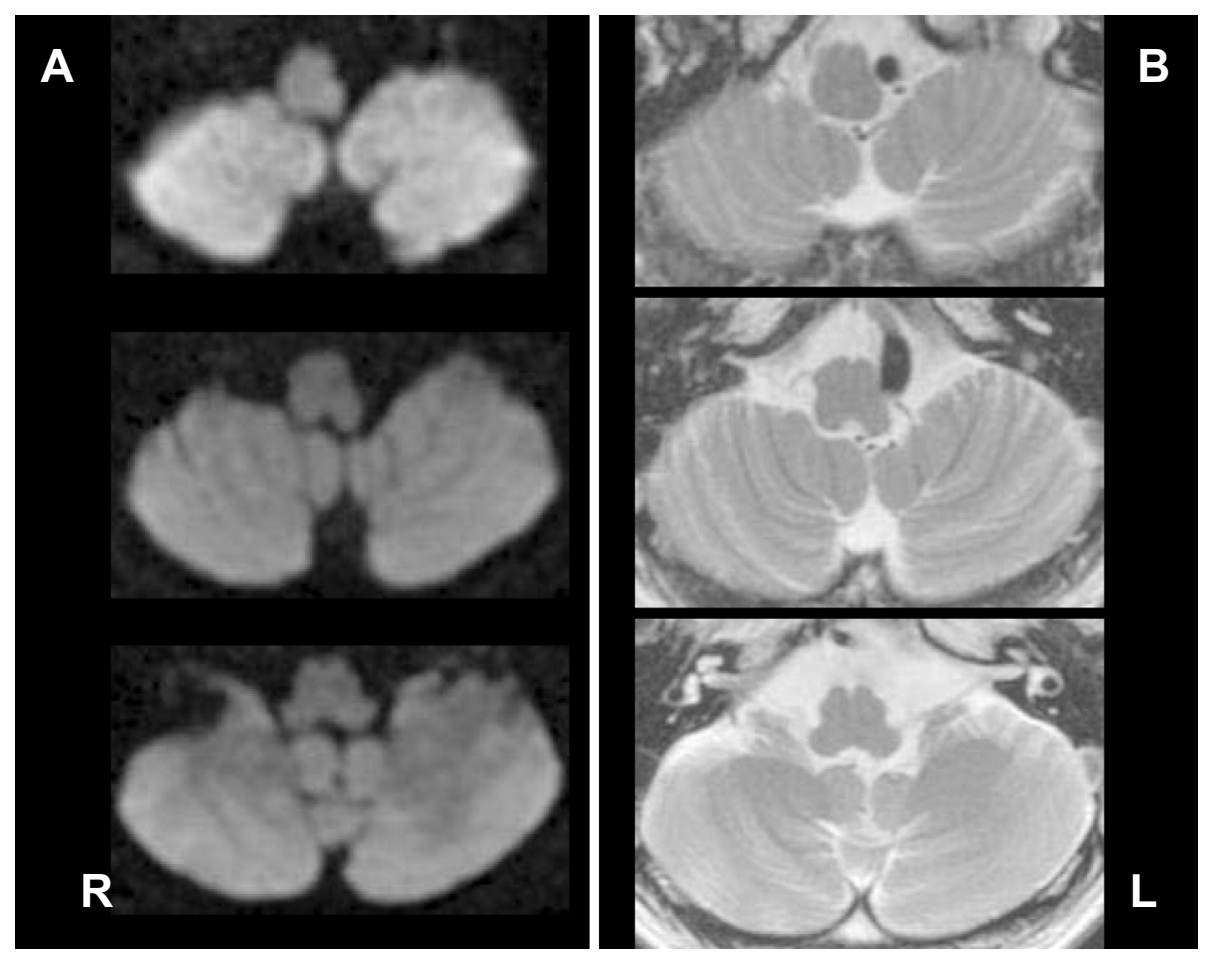

Figure 3. A. Magnetic resonance diffusion-weighted imaging obtained four days after admission revealed no evidence of cerebral infarction. B. T2-weighted magnetic resonance imaging obtained four days after admission revealed severe vascular compression of the left ventrolateral side of the lower medulla oblongata by the left vertebral artery. The medulla oblongata was kidney-shaped, with a deep indentation on the left side.

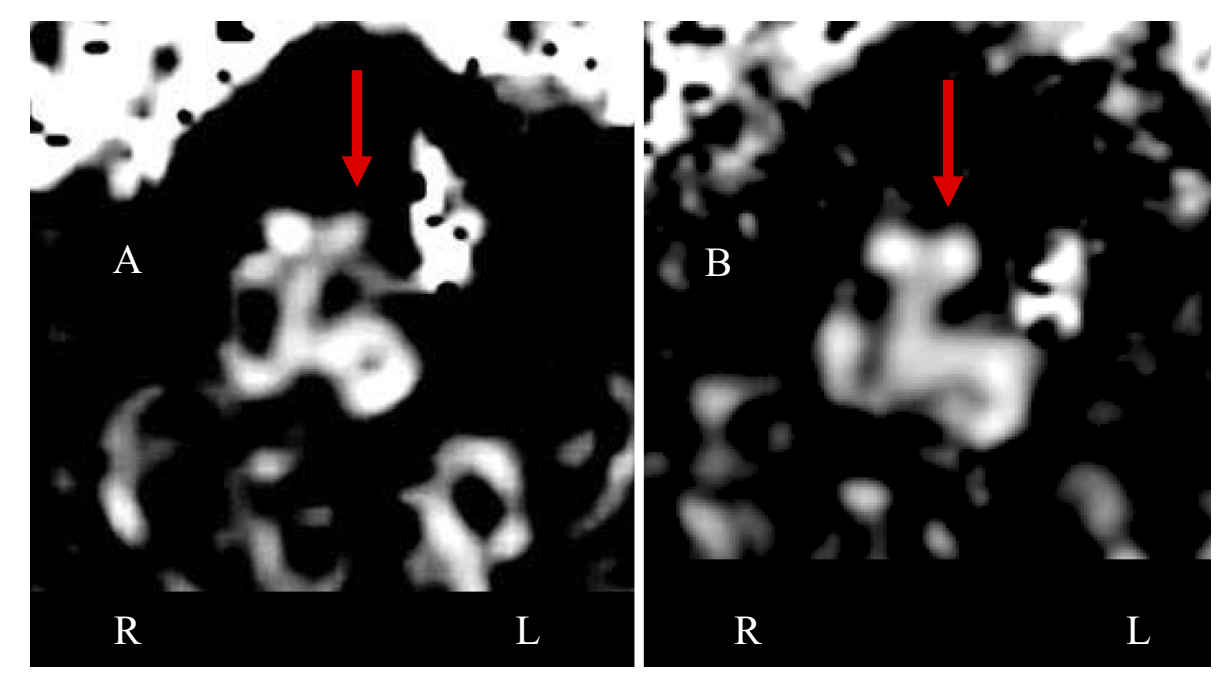

Figure 4. Fractional anisotropy (FA) map created by diffusion tensor imaging. A. Four days after admission, when the patient presented with left hemiparesis, the FA level in the left corticospinal fibers caudal to the pyramidal decussation (arrow) was decreased compared to that observed in the right corticospinal fibers. B. When the symptoms resolved, the decreased FA level in the left corticospinal fibers improved (arrow).

White matter (such as the nerve fiber-rich pyramidal tract) exhibits intensified anisotrophy and the FA level is approximately 1 . As a result, white matter is visualized as high intensity areas on FA maps. In white matter, anisotrophy arises due to nerve fibers running along a unidirectional course. On the other hand, if the nerve fibers are somehow afflicted, anisotrophy is disturbed and consequently the FA level decreases. This investigative approach suggests the possibility that diffusion tensor imaging can detect white matter lesions with high sensitivity (19). Fujimori et al. (20) stated that low FA values indicate degeneration of the ponto-cerebellar tracts projecting through the middle cere- 


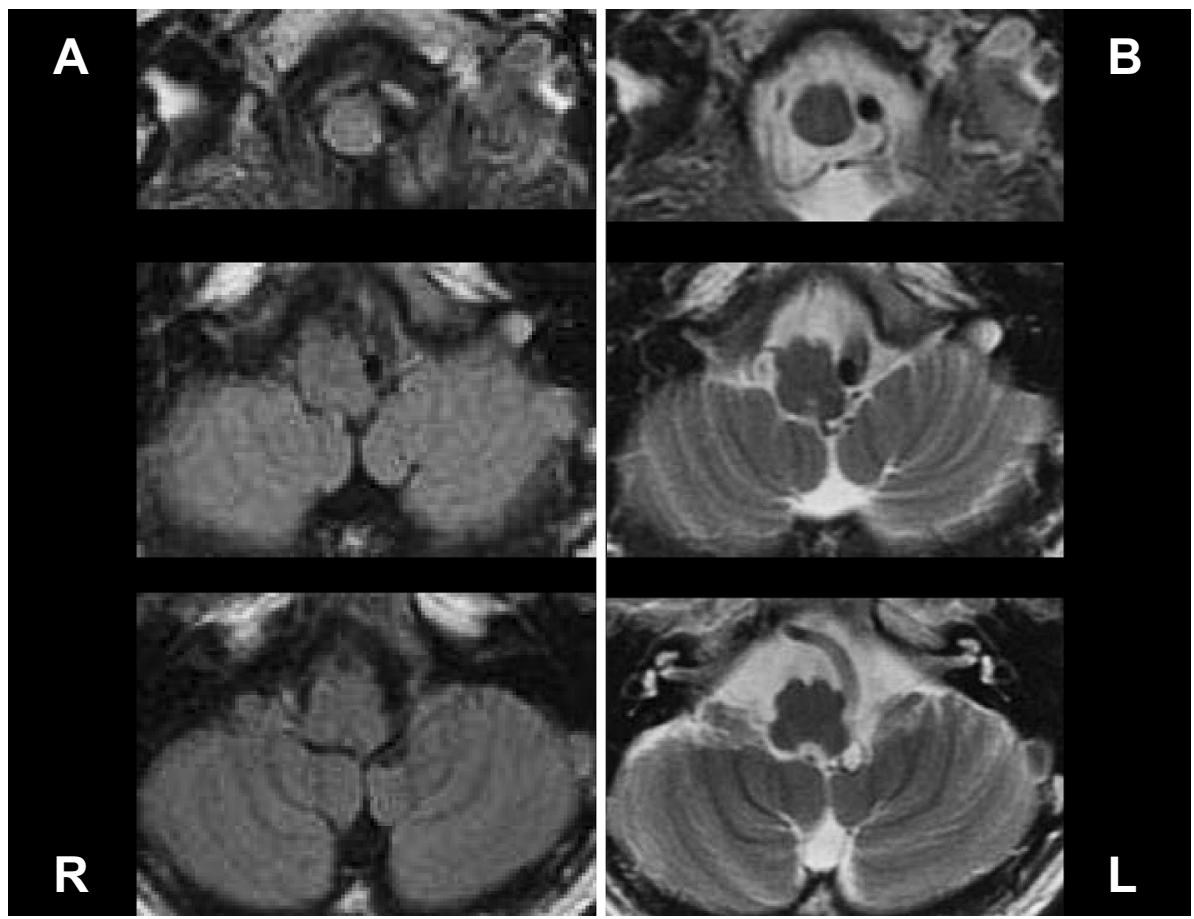

Figure 5. A. MRI with a fluid-attenuated inversion recovery sequence performed in October 2006 (four months after discharge) revealed no evidence of cerebral infarction. B. T2-weighted magnetic resonance imaging obtained in October 2006 revealed no evidence of cerebral infarction, and the degree of vascular compression appeared to be slightly alleviated compared to that previously observed.

bellar peduncle, even in patients with early stage multiple system atrophy. They also stated that visualization of degenerated ponto-cerebellar tracts using diffusion tensor imaging is be useful for early diagnosis. Terajima et al. (21) considered that normal-appearing brain tissue (where conventional MRI does not show abnormal findings) may be responsible for cognitive or locomotor disability in patients with multiple sclerosis and has a crucial influence on the prognosis of that disease. They reported that the FA levels in injured brain tissue that appears normal on conventional MRI were significantly lower in patients with multiple sclerosis than in healthy volunteers. Yin et al. (22) reported that amyotrophic lateral sclerosis patients exhibit significantly lower FA levels in the cortical spinal tract than controls, although conventional MRI often showes normal findings in the early stages of amyotrophic lateral sclerosis. They mentioned that measurement of FA yields the most robust results with regard to axonal degeneration. These reports (19-22) demonstrate that the FA level can be used to evaluate primary and secondary white matter lesions (23). Moreover, in the present case, the fact that the grade of paralysis was slightly more severe in the leg than in the arm may be explained by the topographical distribution of the nerve fibers. These findings suggest that vascular compression of the lateral side of the medulla oblongata was responsible for the patient's hemiparesis.

It has been reported that microvascular compression can be relieved and, consequently, the corresponding symptoms improved following decompression surgery $(12,14-16)$. On the other hand, it has also been reported that one patient's symptoms did not improve with decompression surgery (13). Other authors have reported that patients' symptoms resolved with conservative therapy that did not include surgery and mentioned that a surgical approach should be very cautiously considered $(17,18)$. In the present case, the patient's symptoms gradually improved and resolved within one month without surgical therapy and did not recur with antihypertensive therapy. Furthermore, since discharge, he has not required any antihypertensive medications due to a spontaneous reduction of blood pressure. It was suspected that the cause of vascular compression in the present case was VA dissection. Nagahata et al. (24) discussed the temporal course of unruptured VA dissection. The dissected segment always looked like a fusiform aneurysm on basiparallel anatomical scanning (BPAS)-MRI in their series. Fusiform dilatation of the vascular surface should be a definitive finding for the diagnosis of VA dissection because the inner luminal findings of acute VA dissection on MRA or digital subtraction angiography (DSA) are variable. In addition, resolution of fusiform dilatation on BPAS-MRI should be a sign of healing of VA dissection. Because the VA dissection gradually improved and the degree of compression was consequently less serious, the patient's symptoms might have spontaneously improved.

Recent progress in diagnostic imaging technologies such as MRI and MRA has enabled the observation that compression of the medulla oblongata by a tortuous and dolichoec- 
tatic VA is not a rare occurrence. Interestingly, the pathological significance is not frequent in such cases. For patients who present with brainstem dysfunction, the relationship between clinical symptoms and diagnostic imaging findings should be carefully considered. We believe that FA mapping is a useful clinical tool for evaluating and proving the causal relationship between imaging findings and clinical symptoms.

The authors state that they have no Conflict of Interest (COI).

\section{References}

1. Opalski A. Un nouveau syndrome sous-bulbaire partiell' artere vertebro-spinal posterieure. Paris Med 1: 214-220, 1946.

2. Garcia-Garcia J, Ayo-Martín O, Segura T. Lateral medullary syndrome and ipsilateral hemiplegia (Opalski syndrome) due to left vertebral artery dissection. Arch Neurol 66: 1574-1575, 2009.

3. Kimura Y, Hashimoto H, Tagaya M, Abe Y, Etani H. Ipsilateral hemiplegia in a lateral medullary infarct-Opalski's syndrome. J Neuroimaging 13: 83-84, 2003.

4. Montaner J, A'lvarez-Sabin J. Opalski's syndrome. J Neurol Neurosurg Psychiatry 67: 688-689, 1999.

5. Dhamoon SK, Iqbal J, Collins GH. Ipsilateral hemiplegia and the Wallenberg syndrome. Arch Neurol 41: 179-180, 1984.

6. Liu CY, Chang FC, Hu HH, Hsu LC. Ipsilateral crural monoparesis in lateral medullary infarction due to vertebral artery dissection. Eur J Neurol 13: e8-e9, 2006.

7. Levy M, Levy E, Maimon S. Atypical postpartum stroke presenting as Opalski syndrome: case report and review of the literature. Case Rep Neurol 3: 191-198, 2011.

8. Hermann DM, Jung HH, Bassetti CL. Lateral medullary infarct with alternating and dissociated sensorimotor deficits: Opalski's syndrome revisited. Eur J Neurol 16: e72-e74, 2009.

9. Nakamura S, Kitami M, Furukawa Y. Opalski syndrome: Ipsilateral hemiplegia due to a lateral-medullary infarction. Neurology 75: $1658,2010$.

10. Bailon O, Garcia PY, Logak M, Timsit S. Opalski syndrome detected on DWI MRI: A rare lateral medullary infarction. Case report and review. Rev Neurol (Paris) 167: 177-180, 2011.

11. Cheng MY, Sung CY. Opalski Syndrome. Acta Neurologica Taiwanica 14: 162-163, 2005.

12. Kim P, Ishijima B, Takahashi H, Shimizu H, Yokochi M. Hemiparesis caused by vertebral artery compression of the medulla ob- longata. J Neurosurg 62: 425-429, 1985.

13. Hongo K, Kobayashi S, Hokama M, Sugita K. Vertebral artery section for treating arterial compression of the medulla oblongata. J Neurosurg 79: 116-118, 1993.

14. Murata H, Waga S, Kojima T, Shimizu T, Shimizu S. Medulla oblongata compression by tortuous vertebral artery: case report. No Shinkei Geka 23: 349-353, 1995 (in Japanese, Abstract in English).

15. Hongo K, Nakagawa $\mathrm{H}$, Morota $\mathrm{N}$, Isobe $\mathrm{M}$. Vascular compression of the medulla oblongata by the vertebral artery: report of two cases. Neurosurgery 45: 907-910, 1999.

16. Salvi F, Mascalchi M, Bortolotti C, et al. Hypertension, hyperekplexia, and pyramidal paresis due to vascular compression of the medulla. Neurology 55: 1381-1384, 2000.

17. Iwasaki $Y$, Nakamura $T$, Hamada $K$. Hypoalgesia and hypothermesthesia in a lower extremity due to compression of the medulla oblongata by an elongated vertebral artery. Rinsho Shinkeigaku 44: 176-181, 2004 (in Japanese, Abstract in English).

18. Ishikawa $T$, Nagayama $M$, Iida $M$, Shinohara $Y$. Bulbovascular compression by megadolichobasilar artery manifested as neurogenic and refractory hypertension. Rinsho Shinkeigaku 44: 359364, 2004 (in Japanese, Abstract in English).

19. Matsui N, Nakane S, Harada M, et al. Neuroradiological study of a possible progressive multifocal leukoencephalopathy using diffusion tensor imaging and proton magnetic resonance spectroscopy. Rinsho Shinkeigaku 46: 555-560, 2006 (in Japanese, Abstract in English).

20. Fujimori J, Tatewaki Y, Shimizu H, Kimura I, Hisanaga K. Degeneration of ponto-cerebellar tract visualized by diffusion tensor imaging in multiple system atrophy. Rinsho Shinkeigaku 51: 271274, 2011 (in Japanese, Abstract in English).

21. Terajima $K$, Matsuzawa $H$, Tanaka $K$, Nishizawa $M$, Nakada T. Cell-oriented analysis in vivo using diffusion tensor imaging for normal-appearing brain tissue in multiple sclerosis. NeuroImage 37: 1278-1285, 2007.

22. Yin H, Lim CCT, Ma L, et al. Combined MR spectroscopic imaging and diffusion tensor MRI visualizes corticospinal tract degeneration in amyotrophic lateral sclerosis. J Neurol 251: 1249-1254, 2004.

23. Kondo M, Watanabe-Hosomi A. Clinical application of diffusion tensor imaging: evaluation of aphasia. Higher Brain Function Research 30: 398-403, 2010 (in Japanese, Abstract in English).

24. Nagahata M, Manabe H, Hasegawa S, Takemura A. Morphological change of unruptured vertebral artery dissection on serial MR examinations. Evaluation of the arterial outer contour by basiparallel anatomical scanning (BPAS)-MRI. Interv Neuroradiol 12: 133-136, 2006.

(C) 2013 The Japanese Society of Internal Medicine http://www.naika.or.jp/imonline/index.html 